\title{
DEPFET detectors for direct detection of MeV Dark Matter particles
}

\author{
A. Bähr ${ }^{1}$, H. Kluck ${ }^{2,3}$, J. Ninkovic ${ }^{1}$, J. Schieck ${ }^{2,3, a} \mathbb{D}$, J. Treis ${ }^{1}$ \\ ${ }^{1}$ Max-Planck-Gesellschaft Halbleiterlabor, Otto Hahn Ring 6, 81739 Munich, Germany \\ ${ }^{2}$ Institut für Hochenergiephysik der Österreichischen Akademie der Wissenschaften, Nikolsdorfer Gasse 18, 1050 Vienna, Austria \\ ${ }^{3}$ Atominstitut, Technische Universität Wien, Stadionallee 2, 1020 Vienna, Austria
}

Received: 8 June 2017 / Accepted: 18 December 2017 / Published online: 26 December 2017

(C) The Author(s) 2017. This article is an open access publication

\begin{abstract}
The existence of dark matter is undisputed, while the nature of it is still unknown. Explaining dark matter with the existence of a new unobserved particle is among the most promising possible solutions. Recently dark matter candidates in the $\mathrm{MeV}$ mass region received more and more interest. In comparison to the mass region between a few $\mathrm{GeV}$ to several $\mathrm{TeV}$, this region is experimentally largely unexplored. We discuss the application of a RNDR DEPFET semiconductor detector for direct searches for dark matter in the $\mathrm{MeV}$ mass region. We present the working principle of the RNDR DEPFET devices and review the performance obtained by previously performed prototype measurements. The future potential of the technology as dark matter detector is discussed and the sensitivity for $\mathrm{MeV}$ dark matter detection with RNDR DEPFET sensors is presented. Under the assumption of six background events in the region of interest and an exposure of $1 \mathrm{~kg}$ year a sensitivity of about $\bar{\sigma}_{e}=10^{-41} \mathrm{~cm}^{2}$ for dark matter particles with a mass of $10 \mathrm{MeV}$ can be reached.
\end{abstract}

\section{Introduction}

Several independent measurements clearly point towards the existence of dark matter. The nature of dark matter is still not understood and is among the biggest outstanding problems of modern physics [1]. A well motivated solution to this problem is the existence of a new particle candidate, which interacts at most weakly with standard model particles. The possible mass range of this particle candidate, as well as the possible interaction strength with ordinary matter, spans several orders of magnitude [2]. Recently several theoretical studies focus on possible dark matter candidates in the $\mathrm{MeV}$ mass region, below the mass scale of weakly interacting massive particles [3-8]. This mass region is experimentally

\footnotetext{
a e-mail: Jochen.Schieck@oeaw.ac.at
}

less explored and opens a large space for undiscovered dark matter candidates.

Direct detection experiments search for relic dark matter particles by looking for elastic scatterings between a dark matter candidate and a nucleus. The energy deposited in the scattering processes, the nuclear recoil-energy, can be measured by the experiment. By using simple kinematic relations the mass of the dark matter particle can be inferred from the recoil energy. The sensitivity towards low mass dark matter particles is determined by the detection threshold for nuclear recoils. Dark matter candidates with masses below $100 \mathrm{MeV}$ lead to a nuclear recoil as low as a few $\mathrm{eV}$ and are therefore below the threshold of direct dark matter detection experiments. The search for light dark matter particles via scattering with an electron opens the opportunity to extend the reach towards even smaller masses, down to a few $\mathrm{MeV}$. However, the theoretical prediction of the dark matter-electron scattering rate is more complex, compared to the nuclear scattering. In this paper we study the possibility to measure dark matter-electron scattering using a silicon based detector. In solid state detectors electrons are bound to the nucleus and can no longer being considered as free particles. Electrons are not at rest and the typical speed is greater compared to the average speed of the dark matter particle, leading to a different kinematics of the process. In addition the complicated electronic structure of the semiconductor makes the calculation of the scattering rate more complicated. This topic has been discussed in detail in the literature, e.g. in [9-12], and is only summarised here.

A semiconductor detector based on the DEPFET principle (DEpleted P-channel Field Effect Transistor) with repetitive non-destructive readout (RNDR) [13] offers the possibility to perform a low-noise measurement of the ionisation signal originating from a dark matter-electron inelastic scattering process, down to a single electron. The excellent noise performance for the ionisation signal is reached by repeating 
the measurement in a statistically independent way. With the average ionisation energy for a single electron of a few $\mathrm{eV}$ the detector performance can be transformed to a sensitivity for dark matter masses down to a few MeV.

We briefly review the detection of $\mathrm{MeV}$ dark matter with semiconductor targets in Sect. 2, the RNDR DEPFET detector principle and the expected detector performance is discussed in Sect. 3 and in Sect. 4 we present the expected sensitivity for $\mathrm{MeV}$ direct dark matter detection. In Sect. 5 we summarise the potential of RNDR DEPFET detectors for direct dark matter detection.

\section{Detection of $\mathrm{MeV}$ dark matter by dark matter-electron scattering}

The process of dark matter-electron scattering is derived and discussed in references [9-12]. In this section we summarise the key findings of [10], which are necessary to discuss the expected sensitivity for RNDR DEPFET dark matter detectors in Sect. 4. The reader is referred to [10] for a the complete derivation, in particular about the crystal form factor of silicon, which contains relevant information about the electron binding in the corresponding material.

The measurement of the recoil energy distribution from the dark matter scattering process, together with the expected velocity distribution of the dark matter gives an estimate of the mass of the incoming dark matter particle. For dark matter-nucleus scattering the mass can be derived by simple kinematic calculations and the deposited recoil energy is proportional to $\frac{1}{m_{\mathrm{N}}}$, with $m_{\mathrm{N}}$ being the mass of the target nucleus. A lighter target material therefore returns an increased average recoil energy, which is experimentally easier to measure. The scattering between a dark matter particle and an electron is more complicated and requires a careful discussion. Compared to the dark matter-nucleus scattering no simple interpretation of the scattering rate in terms of cross-section and dark matter mass is possible. Two points are discussed in order to understand the relation between the dark matter scattering rate and the underlying dark matter parameters: the kinematic relation of the scattering process of $\mathrm{MeV}$ dark matter particles and the relevant binding effects of electrons in silicon. In a solid state device made of silicon electrons are bound and cannot be considered to be at rest. The energy transferred to the electron $E_{\mathrm{e}}$ can be derived from a simple energy conservation relation $E_{\mathrm{e}}=-\Delta E_{\chi}-E_{\mathrm{N}}$ [10], with $\Delta E_{\chi}$ being the energy loss of the dark matter particle and $E_{\mathrm{N}}$ being the recoil energy of the whole atom. Please note that the energy $E_{\mathrm{e}}$ is the total energy and only parts of the energy is finally transferred as the kinetic energy of the electron, while the rest is needed to move the electron from the valence band to the conduction band. We consider small energy transfers only and therefore the recoil energy of the atom, $E_{\mathrm{N}}$, can be safely set to zero. The average velocity of the electron can be related to its binding energy, $\mathrm{v}_{\mathrm{e}} \sim Z_{\text {eff }} \alpha$, with $\alpha \approx 1 / 137$ being the fine-structure constant and the effective charge of the nucleus $Z_{\text {eff }}$ being one for outer electrons. The velocity $\mathrm{v}_{\mathrm{e}}$ is large compared to the velocity of the incoming dark matter particle, $\mathrm{v} / c \sim 10^{-3}$. The average momentum transfer of the scattering process is therefore dominated by the momentum of the bound electron. This information, together with the energy conservation relation, can be used to show that the typical available momentum transfer $q$ in $\mathrm{MeV}$ dark matter scatterings is enough to move electrons from the valence band to the conduction band of silicon, with a band gap in the order of a few eV.

Parts of the energy transferred from the dark matter particle to the electron $E_{\mathrm{e}}$ is needed to move the electron from the valence band to the conductance band. To predict the electron scattering rate the relevant electron binding effects for silicon need to be calculated. The calculation of a dimensionless crystal form factor $f_{\text {crystal }}\left(q, E_{\mathrm{e}}\right)$ was performed for the first time in [10] and can be considered as a key input to the prediction of the dark matter-electron scattering rate in silicon. The form factor calculation implies that the scattering processes with larger $q$ values are suppressed compared to processes with low $q$, leading to a sensitivity increase towards low energy recoils. The differential recoil rate can be written as [10]:

$$
\begin{aligned}
\frac{d R}{d \ln E_{\mathrm{e}}}= & \frac{\rho_{\chi}}{m_{\chi}} N_{\text {cell }} \bar{\sigma}_{\mathrm{e}} \alpha \frac{m_{\mathrm{e}}^{2}}{\mu_{\chi \mathrm{e}}^{2}} \\
& \times \int d \ln q\left(\frac{E_{\mathrm{e}}}{q} \eta\left(v_{\min }\left(q, E_{\mathrm{e}}\right)\right)\right) \\
& \times F_{\mathrm{DM}}(q)^{2}\left|f_{\text {crystal }}\left(q, E_{\mathrm{e}}\right)\right|^{2},
\end{aligned}
$$

with $\rho_{\chi}$ being the local dark matter density, $m_{\chi}$ the mass of the dark matter particle, $N_{\text {cell }}$ the number of unit cells in the target, $\bar{\sigma}_{\mathrm{e}}$ parametrizing the strength of the interaction, $m_{\mathrm{e}}$ the mass of the electron, $\mu_{\chi} \mathrm{e}$ the reduced mass of the dark matterelectron system and $\eta\left(v_{\min }\left(q, E_{\mathrm{e}}\right)\right)$ parametrizing the dark matter density profile. The dark matter form factor $F_{\mathrm{DM}}(q)$ parametrises the momentum dependence of the interaction. For $F_{\mathrm{DM}}(q)=1$ the interaction strength $\bar{\sigma}_{\mathrm{e}}$ is reduced to a simple point like interaction. $F_{\mathrm{DM}}(q)=\left(\alpha m_{\mathrm{e}} / q\right)$ corresponds to an electric dipole moment and $F_{\mathrm{DM}}(q)=$ $\left(\alpha m_{\mathrm{e}} / q\right)^{2}$ corresponds to the exchange of a massless (or ultra-light) vector mediator. For our studies we choose the simplest momentum dependency and we set $F_{\mathrm{DM}}(q)=1$, as expected for a point-like interaction.

The energy deposited via the dark matter scattering process $E_{\mathrm{e}}$ is converted to an average number of produced electrons $Q$, by setting the average ionisation energy to $E_{\text {ion }}=3.6 \mathrm{eV}$ and the band-gap energy to $E_{\text {gap }}=1.11 \mathrm{eV}$. The ionization $Q$ is given by 


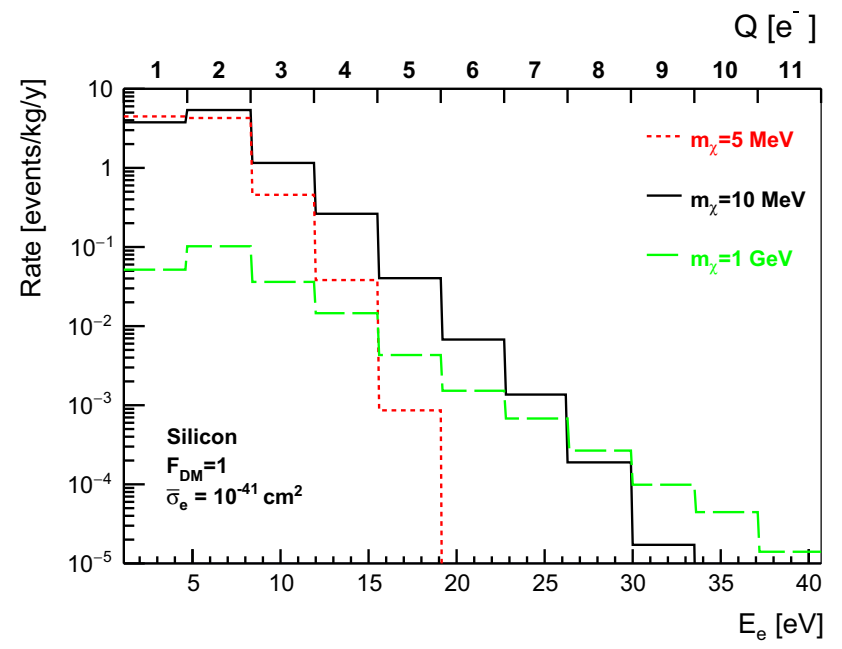

Fig. 1 The expected recoil spectrum for a non-relativistic dark matter elastic cross-section of $\bar{\sigma}_{\mathrm{e}}=10^{-41} \mathrm{~cm}^{2}$ as a function of the deposited energy $E_{\mathrm{e}}$ and the ionization $Q$. The rate is shown for incoming dark matter particles with a mass of $1 \mathrm{GeV}$ (green, dashed), $10 \mathrm{MeV}$ (black, solid) and $5 \mathrm{MeV}$ (red, dotted). We assume standard astrophysical assumptions for the dark matter density and velocity distribution (The dark matter density is set to $0.4 \mathrm{GeV} / \mathrm{cm}^{3}$, a Maxwell-Boltzmann velocity distribution for the dark matter particles is assumed with $300 \mathrm{~km} / \mathrm{s}$ as mean velocity and the escape velocity is set to $600 \mathrm{~km} / \mathrm{s}$.) The form factor $\mathrm{F}_{\mathrm{DM}}$ is set to one

$Q\left(E_{\mathrm{e}}\right)=1+\operatorname{Int}\left[\left(E_{\mathrm{e}}-E_{\mathrm{gap}}\right) / E_{\mathrm{ion}}\right]$,

[10]. The expected recoil rate as a function of deposited energy $E_{\mathrm{e}}$ is shown in Fig. 1 . For a $10 \mathrm{MeV}$ dark matter particle about $65 \%$ of all events generate at least two electrons in the detector. The rate is calculated by using the publicly available QEdark code [10]. ${ }^{1}$ The expected sensitivity is presented after discussing the expected performance of the RNDR DEPFET device in Sect. 4 in terms of detected electrons.

\section{RNDR DEPFET sensors for direct dark matter detection}

\subsection{Concept of RNDR DEPFET devices}

The basic idea behind repetitive non-destructive readout (RNDR) is to apply one of the most important implications of the central limit theorem on the field of detectors. Any charge generated in the sensitive detector bulk is collected in the internal gates. Due to the excellent charge carrier lifetime, charge loss can be virtually excluded. The RMS noise of a single measurement is determined by the electronic noise of the transistor current measurement. By repetitively measuring the identical signal charge in a statistically independent

\footnotetext{
${ }^{1}$ http://ddldm.physics.sunysb.edu/ddlDM/
}

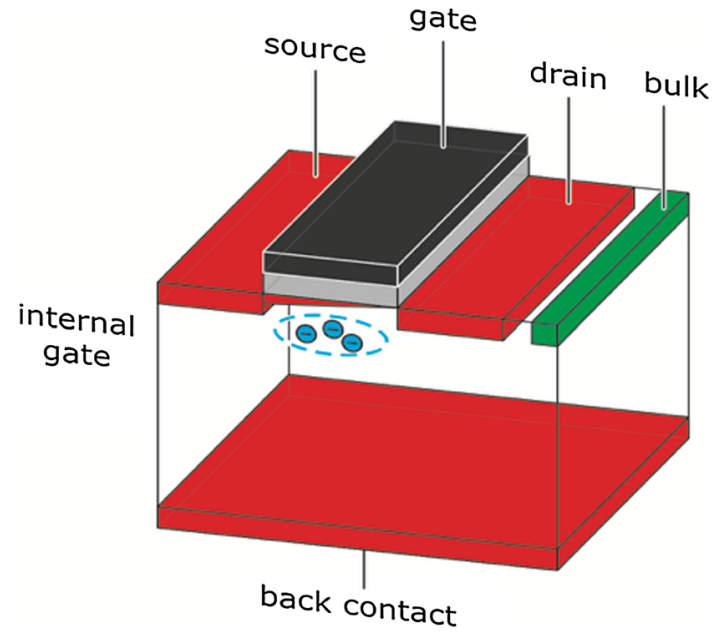

Fig. 2 Structure of a basic DEPFET cell

way, the value resulting from the average of the individual measurements has a standard deviation of $\sigma_{\text {eff }}=\frac{\sigma}{\sqrt{n}}$, with $\sigma$ being the RMS noise of a single measurement, and $n$ being the number of readings. In this way, the standard deviation of the mean can be considered to be the effective noise of the measurement.

Devices based on the combined detector-amplifier structure DEPFET are applied for a variety of particle physics and astrophysical experiments [14-16]. In their most simple form, they provide an active pixel sensor with pixelindividual charge storage and readout at high speed with very good signal-to-noise ratio (SNR). In addition, however, they provide an ideal platform to realise the RNDR principle for radiation detectors.

The simplest DEPFET cell [13] consists of a P-channel FET integrated on a silicon bulk, which is fully depleted by means of sidewards depletion (see Fig. 2). By an additional deep$\mathrm{n}$ implant directly below the gate, a potential minimum for electrons is created, which all bulk-generated electrons will drift to. In case a transistor current is present, their presence modulates the conductivity of the transistor channel, and this modulation is detected by appropriate subsequent electronics. Hereby, the potential minimum has the same effect on the channel as the external gate, and it is therefore also referred to as internal gate. High-accuracy measurements rely on correlated double-sampling (CDS) to determine the amount of charge. After an initial measurement of the transistor state, the charge is removed from the internal gate by an attached $\mathrm{n}$-channel MOSFET, the ClearFET, and the transistor state is measured again with empty internal gate. The actual amount of charge can be precisely determined by the difference. In this way, standard DEPFET cells in circular geometry (see Fig. 3) have been operated with an equivalent noise charge (ENC) of $4-5 \mathrm{e}^{-}$RMS for a readout time of $4 \mu \mathrm{s}$ [17].

The fact, however, that the quantity of charge is sensed indirectly via the channel conductivity enables an efficient 


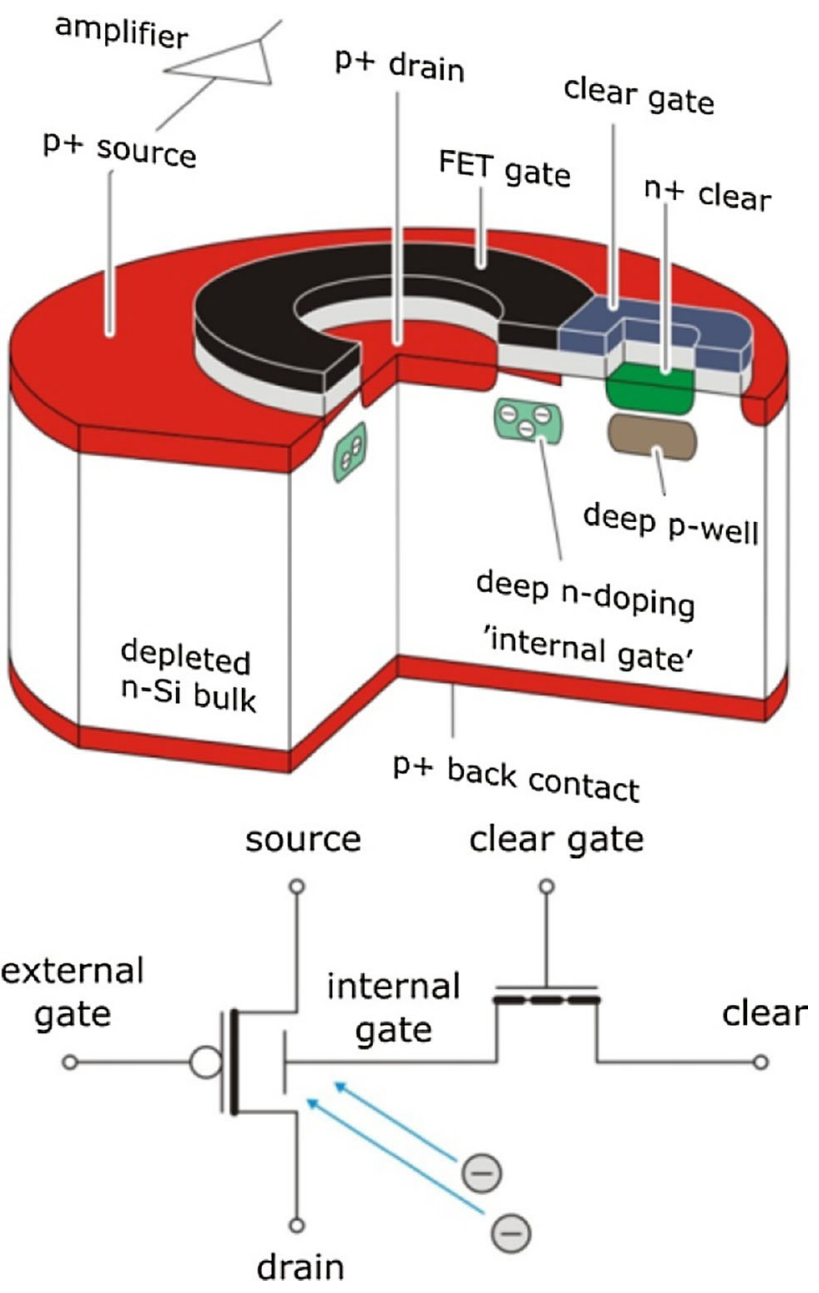

Fig. 3 Structure (top) and circuit representation (down) of a conventional spectroscopy-grade DEPFET cell

implementation of a DEPFET device capable of RNDR. In case the charge is not cleared away during a CDS cycle, but transferred to an adjacent storage node, where the charge is still preserved and where it has also no influence on the DEPFET channel conductivity, mimics a clear process and a nondestructive CDS cycle can be implemented. Transferring the charge back to the DEPFETs internal gate again after the CDS cycle has been finished starts a new CDS cycle for the identical signal charge. In case of the DEPFET, the second storage node can even be the internal gate of a second DEPFET adjacent to the first one, and the transfer can be conducted by means of an additional so-called transfer gate interposed between the two DEPFETs. The second DEPFET can also be used to conduct a CDS measurement, where the clear is replaced by the transfer back to the original DEPFET. This process can be repeated arbitrary times, until the charge is removed by the ClearFET after the final acquisition. In this way, one device pixel can be considered to be a superpixel being composed of two DEPFET subpixels, whose internal

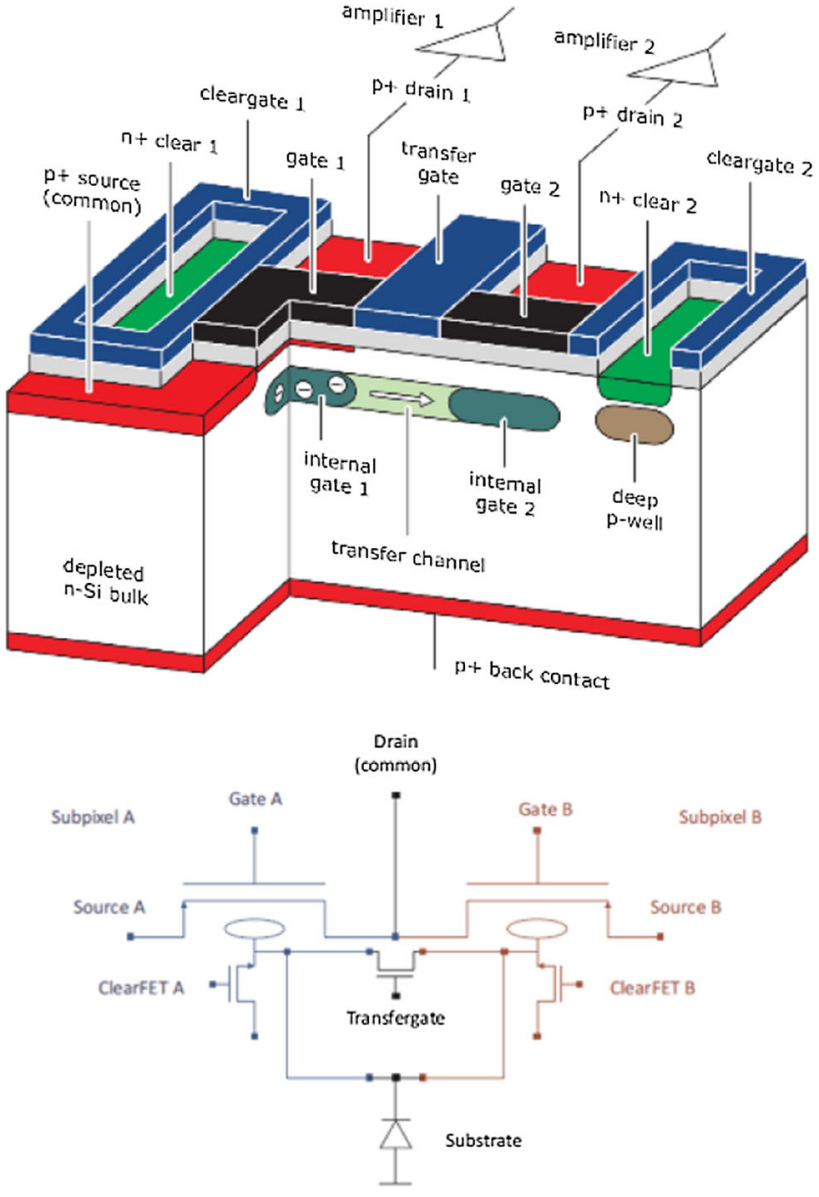

Fig. 4 Structure (top) and circuit representation (bottom) of RNDR DEPFET superpixel consisting of two DEPFET subpixels with linear geometry

gates are connected by the transfer gate. An example for a circuit representation and the respective layout is shown in Fig. 4.

\subsection{Performance model and prototype results}

In practice, however, the RNDR process is disturbed by the advent of additional signal- and leakage electrons from the bulk during the signal evaluation process. This leads to a deviation from the ideal behaviour, which has been described by Bähr's equation [19]:

$\sigma_{\mathrm{eff}}^{2}=\frac{\sigma^{2}}{n}+\Delta \sigma^{2} \cdot\left(\frac{1}{2}+\frac{1}{3} \cdot n-\frac{5}{6} \cdot \frac{1}{n}\right)$

where $\Delta \sigma$ is the expected increase in noise during one CDS acquisition in the RNDR cycle. For given $\Delta \sigma$ and $\sigma$, an optimum number of transfer cycles can be derived:

$n^{\mathrm{opt}}=\sqrt{3 \cdot \frac{\sigma^{2}}{\Delta \sigma^{2}}-\frac{5}{2}}$ 

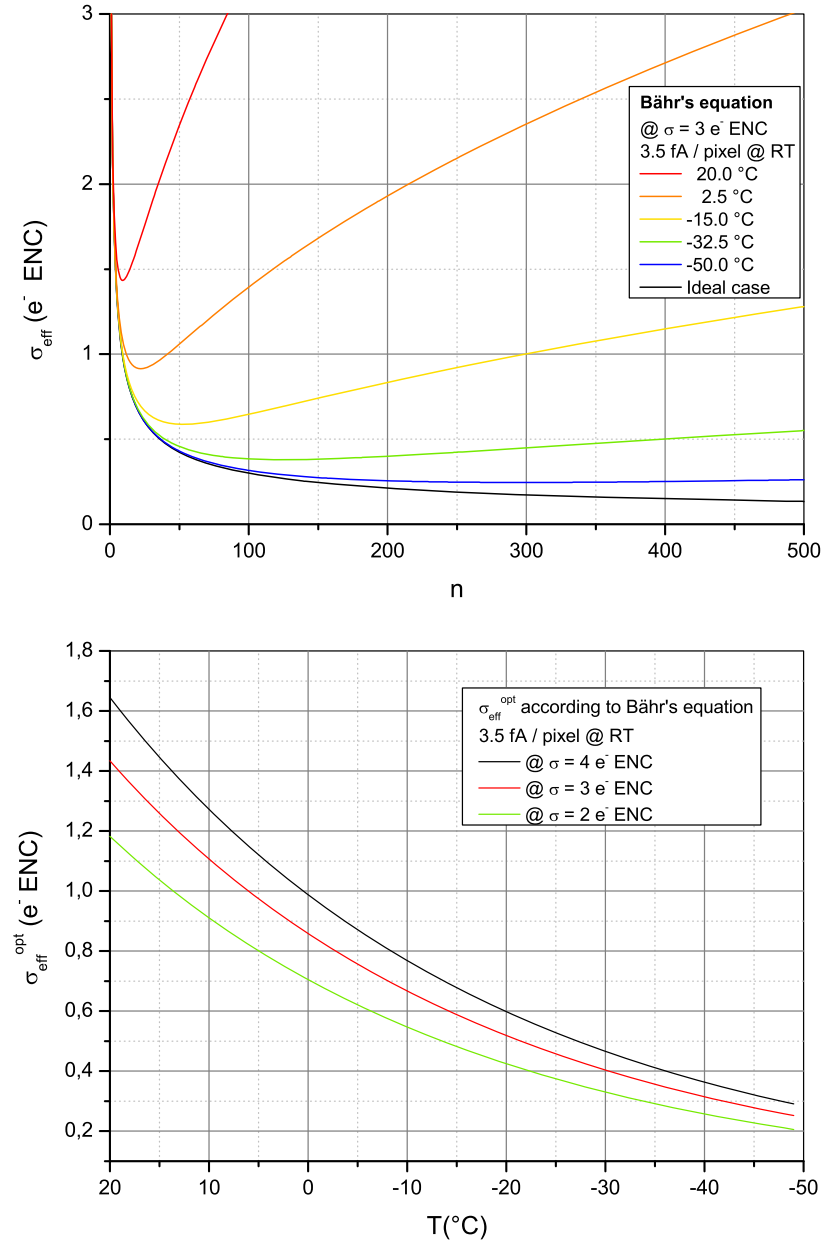

Fig. 5 Example performance graphs according to Bähr's equation: Dependence of $\sigma_{\text {eff }}$ on $n$ and temperature for a pixel with $3 e^{-}$ENC (top) and dependence of $\sigma_{\text {eff }}^{\text {opt }}$ on temperature for three different initial sigma values

resulting in an optimum achievable effective noise of:

$\sigma_{\mathrm{eff}}^{\mathrm{opt}}=\sqrt{\frac{\sigma^{2}}{n^{\mathrm{opt}}}+\Delta \sigma^{2} \cdot\left(\frac{1}{2}+\frac{1}{3} \cdot n^{\mathrm{opt}}-\frac{5}{6} \cdot \frac{1}{n^{\mathrm{opt}}}\right)}$

An example for the dependence can be seen in Fig. 5. The second summand under the square-root in Eq. 5 describes the deviation from the expected $1 / \sqrt{n}$ behaviour due to the influence of the increase in noise $\Delta \sigma$ originating from the leakage current. Inside a silicon detector, this contribution can be efficiently suppressed, but not eliminated, by cooling. For low temperatures, the performance curve will approximate the ideal $1 / \sqrt{n}$ behaviour.

A DEPFET based RNDR device optimised for the detection of the extremely weak signals (i.e. $\sigma<2-3 e^{-}$ENC) can be operated with an optimum number of readout cycles, which allows to lower $\sigma_{\text {eff }}^{\text {opt }}$ down to a level, where the minimum detectable signal (i.e. one electron) can be only gener- ated by noise fluctuations with 5 sigma probability or lower. The application of cumulative measurement techniques (i.e. using the non-destructive readout without clearing of the pixel charge) helps to reduce this source of background (i.e. seeming single electron signals due to noise fluctuations) even further. Here, suppression of the $\Delta \sigma$-contribution in the perturbation term of Bähr's equation to $10^{-4}$, and even lower, helps to achieve a $\sigma_{\text {eff }}^{\text {opt }}$ of $0.2 e^{-}$and below. This is achieved by adopting either the electronic shutter option or the Infinipix topology. Nevertheless, even in case the detector is operated with an effective threshold of one electron, volume leakage current collected during the sensors integration time is a source of irreducible background.

To maintain the sensitivity for the WIMP interaction signature as low as $2-3 e^{-}$, the aim must be to lower the probability of two leakage current electrons within one pixel and frame to as low a level as possible. This can be achieved by operating the device at lowest possible temperatures to decrease the absolute magnitude of the leakage current, or by increasing the readout rate to limit the integration time, or by a combination of both methods. Again, cumulative measurements can help to preserve the statistical significance by preventing performance deterioration due to recombination noise.

Standard mode RNDR DEPFET in circular geometry furnished with compact subpixels sharing the clear contact (see Fig. 6) have been operated in single-pixel and small matrix environments for proof-of-principle measurements and verification of the performance model. Results have been reported in $[18,19]$, some results are shown in Fig. 7. The predictions of Bähr's equation are nicely confirmed by both measurements and Monte Carlo simulations modelling the extended weighting function for the RNDR cycle. The value for $\sigma_{\text {eff }}^{\text {opt }}$ of $0.18 e^{-}$RMS corresponds to the prediction for a device with a value for of $3 e^{-}$at $-50{ }^{\circ} \mathrm{C}$ and 256 transfer cycles. The single electron resolving capability was verified for amounts of charge of up to $10^{3} e^{-}$, the peaks are nicely separated.

The high resistivity float zone silicon used for the fabrication of the sensors has a charge carrier lifetime at room temperature at the order of $1 \mathrm{~s}$. This has to be seen in relation to the drift time in the depleting field, which, depending on the bias voltage, is at the order of 10-20 ns. Cooling of the sensor increases the charge carrier lifetime to levels of minutes, so that an efficiency of $100 \%$ for bulk generated electrons can be assumed with an accuracy of $10^{-9}$. The probability to create an electron-hole pair by the dark matter-electron scattering is fully described by Eq. 1. Highly doped regions on the front- and backside of the sensor, however, can be considered as dead material, reducing the effective mass of the detector and therefore the exposure. The exposure quoted in the sensitivity studies described in Sect. 4 does not include the dead material. The amount of dead material at sensor 


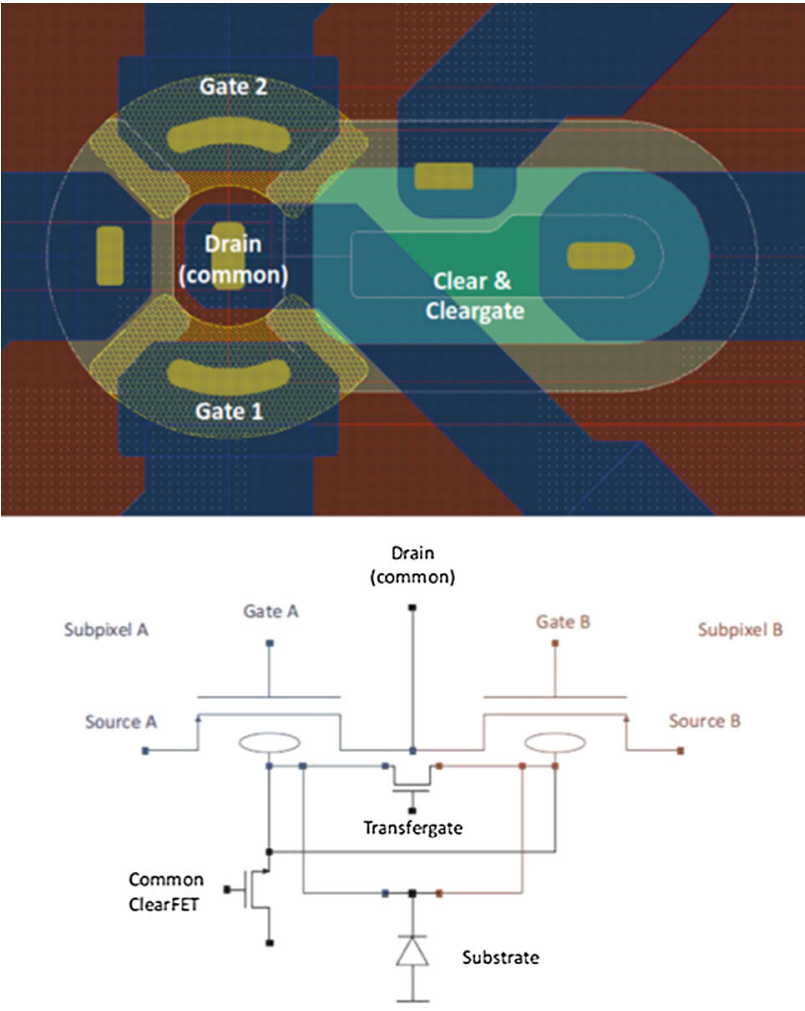

Fig. 6 Compact RNDR DEPFET superpixel layout in circular geometry as operated for the prototype tests (top) and equivalent circuit representation (bottom)

front- and backside, which is expected to be in the range of a few percent, needs to be determined by simulation using the final sensor layout.

\subsection{Planned improvements for future devices}

In addition to the leakage current, a more serious perturbation of the RNDR process arises from the DEPFET's permanent sensitivity. In case signal charge arrives during the RNDR cycle, the signal charge is altered and the resulting mean value of the $n$ measurements does not represent the original signal charge. This is mainly a problem for applications were the incoming radiation is not synchronized with the readout cycle and for the background events for applications where it is. Although running average techniques can be applied during the RNDR process to detect the occurrence of these so-called misfit events, it is better to reduce their overall influence or even to completely avoid it. In this respect, two different approaches have been pursued to optimize RNDRbased detectors for future applications:

- A substantial reduction of the initial noise figure $\sigma$ for a single reading decreases not only $\sigma_{\mathrm{eff}}^{\mathrm{opt}}$ (see Eq. 5), but also
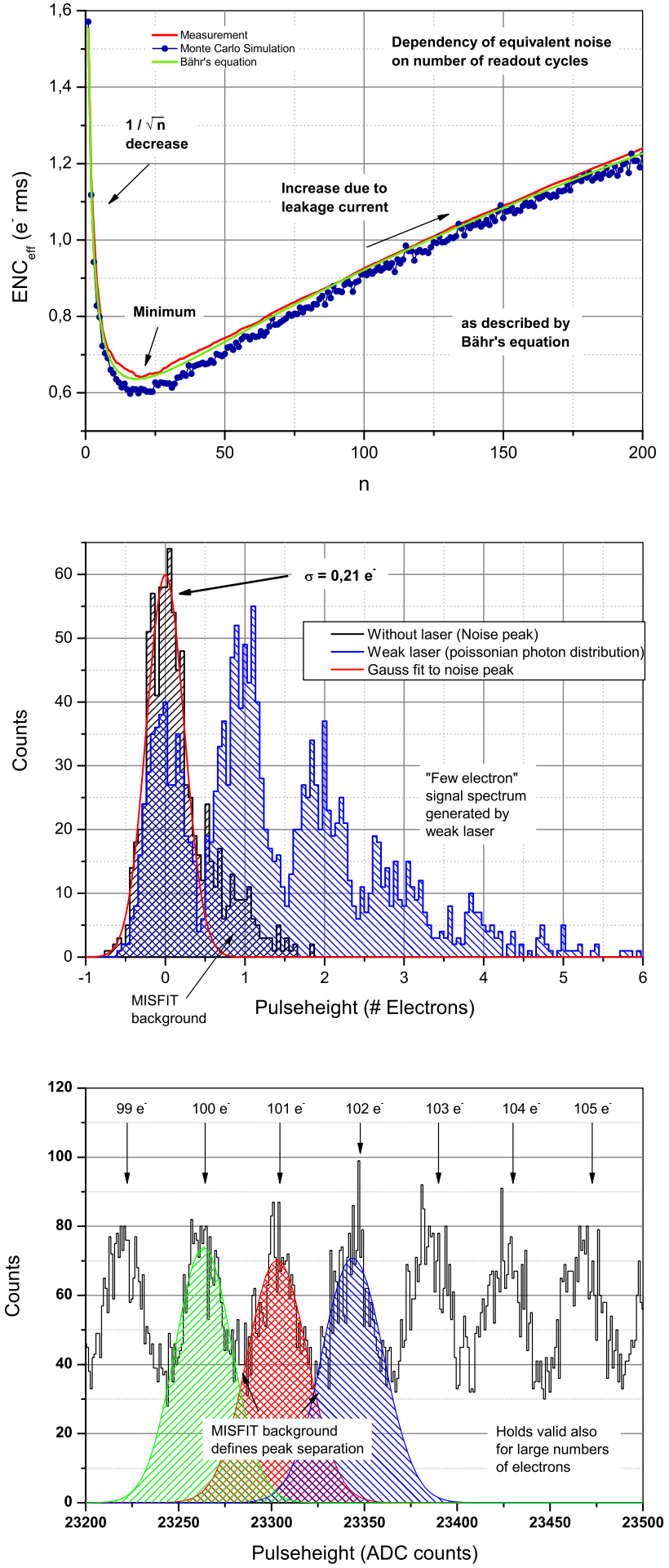

Fig. 7 Example of measurement results for RNDR DEPFET prototypes operated at $-40{ }^{\circ} \mathrm{C}$ : agreement between measured $\sigma_{\text {eff }}$ and $\sigma_{\text {eff }}$ predicted both by Monte Carlo and Bähr's equation (top), single electron spectra taken for weak (middle) illumination intensities exhibiting the expected poisson distribution, and peak separation for higher illumination intensities (bottom). From the distance of the single electron peaks, a $\sigma_{\text {eff }}^{\text {opt }}$ of $0.2 e^{-}$ENC can be derived 
$n^{\text {opt }}$ and, accordingly, the required time for the RNDR cycle. Constant signal rate provided, this in proportion reduces the probability for misfit events.

- In addition, the introduction of a global electronic shutter to the pixel array decouples the DEFET superpixels from the detector bulk. Charge generated in the silicon bulk while the shutter is active will be extracted from the detector volume without being detected. Although this approach introduces some degree of dead-time, it provides a reduction of misfit background by at least two orders of magnitude in addition to the improvements achieved by reducing the noise.

Both options have been evaluated with respect to feasibility. Concerning the first option, an optimization of the DEPFET response by adapting geometry and standard process technology parameters is expected to lower the initial noise figure down to values of $2-1.5 e^{-}$ENC, depending on the shaping time. This lowers both $n^{\text {opt }}$ by an order of magnitude and, accordingly, $\sigma_{\text {eff }}^{\text {opt }}$ to levels far below the single electron threshold [20]. More advanced modifications of the process technology, which are currently under investigation, have the potential to improve the performance even further.

The implementation of an electronic shutter has been evaluated via simulations and on a prototype level, and its functionality has been verified. The introduction of additional blind and blind-gate contacts surrounding the pixel structure allow to extract electrons on demand, providing a charge suppression factor of $10^{-3}$ and higher for the superpixels, while maintaining full retention of charge already stored in the internal gates. The shutter speed is below $100 \mathrm{~ns}$. Figure 8 shows layout and circuit representation of a typical RNDR pixel with shutter functionality.

One of the biggest drawbacks of DEPFET based devices is the pixel size. Current RNDR DEPFET prototype devices exhibit pixel sizes at the order of $75 \times 75 \mu \mathrm{m}^{2}$. This relatively large pixel size is partially counterbalanced by the full depletion in combination with the relatively large device thickness of $450 \mu \mathrm{m}$. Nevertheless, this large pixel size limits the capability for background suppression on the base of cluster analysis especially for events in a very shallow depth beneath the pixel structure. For this reason, compact devices have been designed, which provide for a pixel size of $36 \times 36 \mu \mathrm{m}^{2}$. This very compact design (see Fig. 9) has been realized by combining clear and shutter contacts. The design implements global clear and shutter functionality and allows for incremental as well as absolute charge measurements. In combination with the large bulk thickness, cluster analysis is possible to some extent.

For dark matter detection, arrays of $1 \mathrm{k} \times 1 \mathrm{k}$ of these pixels are proposed covering an area of $\approx 3.7 \times 3.7 \mathrm{~cm}^{2}$, on a fully depleted detector bulk of $1 \mathrm{~mm}$ thickness. Detector

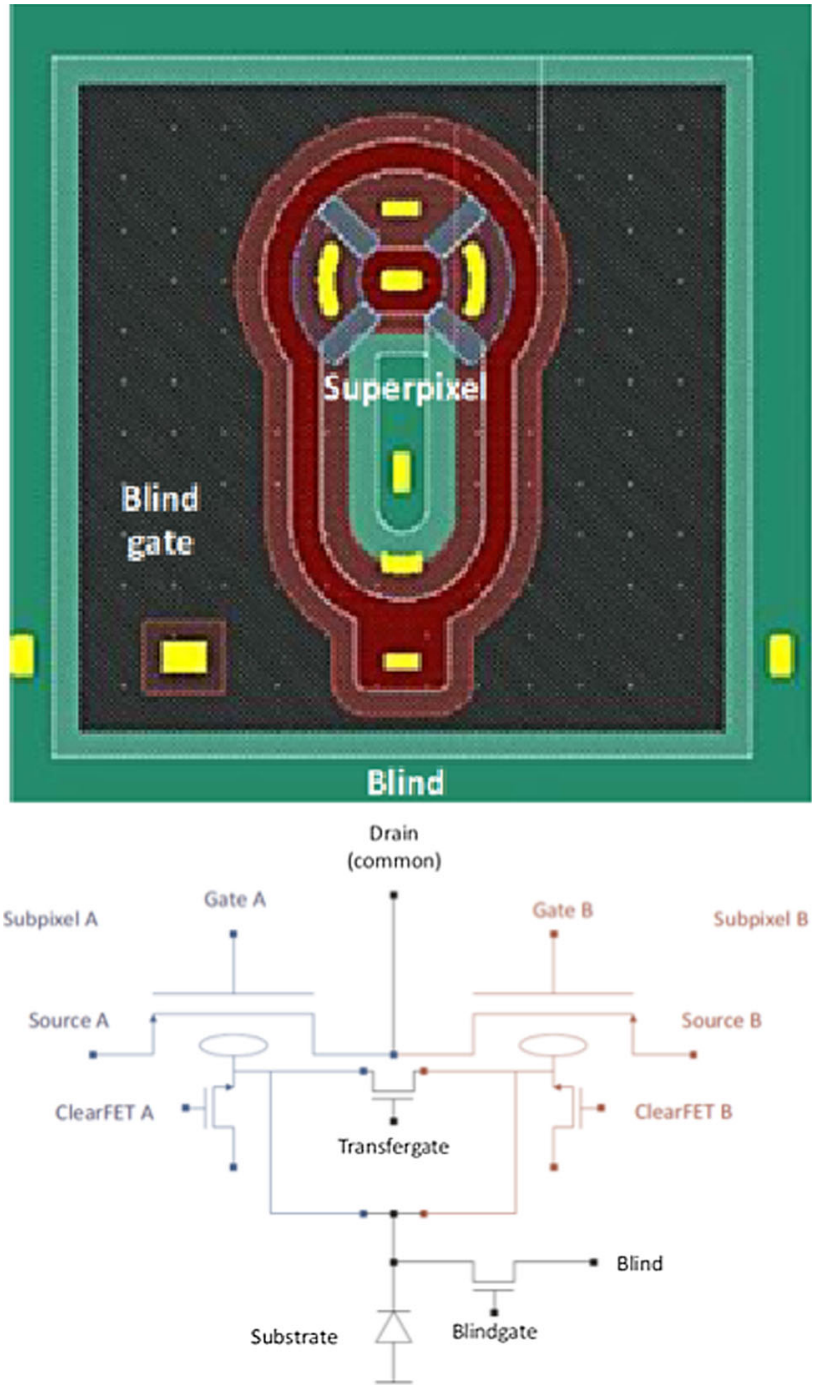

Fig. 8 Layout (top) and equivalent circuit representation (bottom) of RNDR superpixel with electronic shutter. The pixel size here is $75 \times$ $75 \mu \mathrm{m}^{2}$

mass is at the order of $3.2 \mathrm{~g}$. Initial noise is expected to be $1.5 e^{-}$ENC, target noise is $<0.2 e^{-}$ENC.

\subsection{Planned prototype measurements}

The base for the development of such devices will be the data gathered from the upcoming prototype measurements. Here, RNDR DEPFET devices with standard topology with and without global shutter functionality as shown in Figs. 6 and 8 respectively will for the first time be operated on a larger matrix scale in a low background environment. The devices consist of an array of $64 \times 64$ pixels integrated on a $0.45 \mathrm{~mm}$ thick silicon bulk.

Goal of the measurement is the complete parametrization of the devices in terms of operational parameters, operating temperature for lowest leakage current and optimized readout 


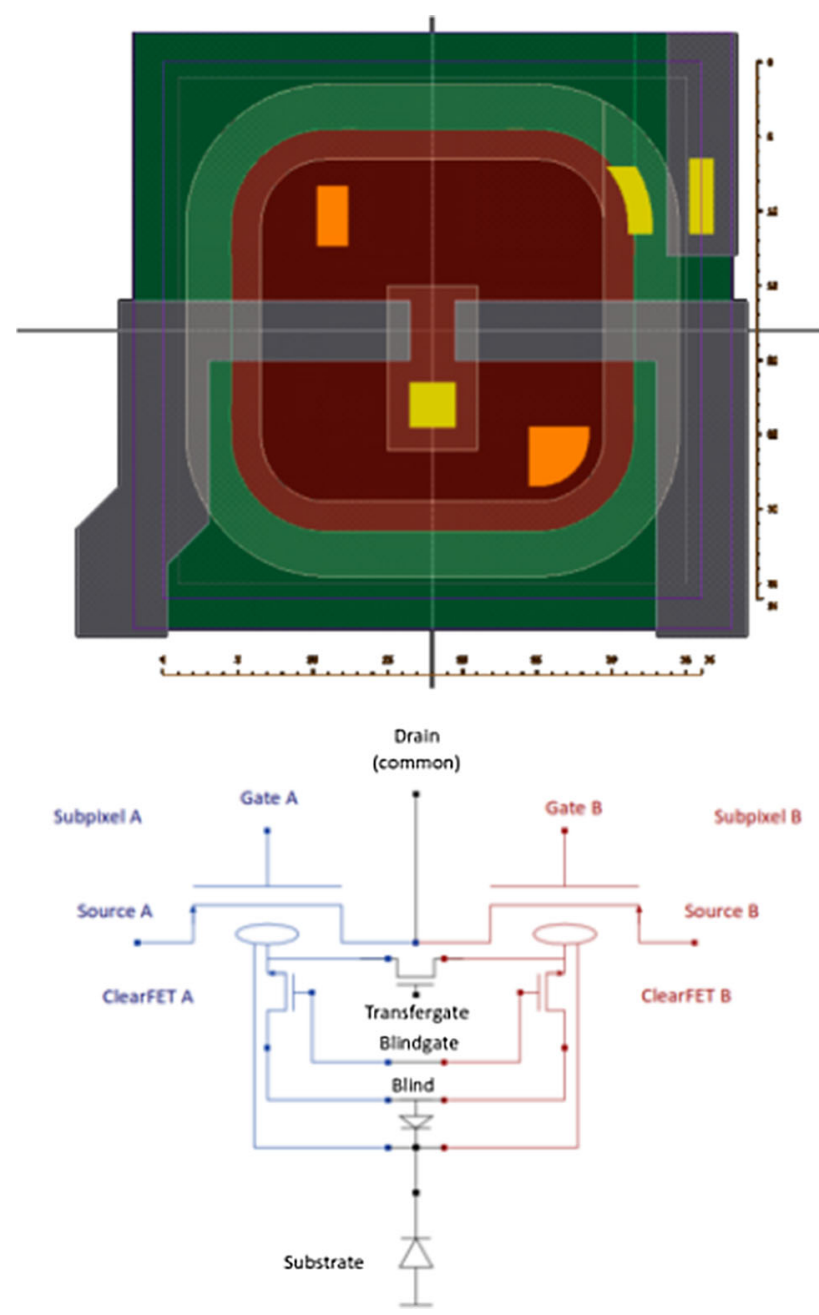

Fig. 9 Layout (top) and equivalent circuit representation (bottom) of RNDR superpixel with electronic shutter. The pixel size here is $36 \times$ $36 \mu \mathrm{m}^{2}$

for optimum noise performance. The readout setup is optimized for background shielding and low noise rather than high speed readout, as the frame rate is at the order of $\mathrm{MHz}$ or even lower.

\section{Dark matter sensitivity studies}

The low-noise measurement performance of the ionisation signal from an inelastic dark matter-electron scattering measured with the RNDR DEPFET sensor described in Sect. 3 can be translated into a sensitivity for the detection of $\mathrm{MeV}$ dark matter. Like in Sect. 2 we use the publicly available QEdark code [10] for the estimate.

We analyse the impact of three key-parameters on the experimental sensitivity to low mass dark matter. Besides the threshold for the ionisation measurement, we study the exposure and the impact of background events on the sen- sitivity. While the DEPFET devices described in Sect. 3.3 have a mass of $3.1 \mathrm{~g}$ only, we will discuss our results with a default exposure of $1 \mathrm{~kg}$ year and presents results with $0.1 \mathrm{~kg}$ year as an alternative scenario. We investigate two main background sources, which could influence the sensitivity: background events caused by the energy depositions from radioactive decays from inside or outside of the experiment and background events generated by the leakage current present during the operation of the silicon sensor. The two background sources have a different impact on the operation of the DEPFET device.

\subsection{Background events from the leakage current}

For the operation of the RNDR DEPFET detector a bias voltage is applied to the sensor. A very small leakage current is generated in the sensor, which can lead to the collection of electrons in the internal gate. These electrons from the leakage current generate background events. The size of the leakage current, and therefore the number of background events, can be reduced by operating the device at lower temperatures.

Even the smallest known leakage current in silicon devices generate a significant background event rate for single pixel hits. The total number of background events from single electron events originating from the leakage current grows proportional to the total exposure time. Any increase of the readout rate of the device will not change the picture. The situation changes for background events with two electrons collected in a single pixel, assuming the probability to generate a single electron from the leakage current is uncorrelated. The probability to collect two electrons from the leakage current in the same pixel is significantly reduced and, in addition, the increase of the readout rate with a regular clear of the internal gates will further reduce the probability to collect two electrons from leakage current in the same pixel. Alternatively, RNDR DEPFET devices allow for cumulative measurements, as the charge within one superpixel does not necessarily have to be cleared, but may remain within the superpixel for later comparative measurements. This can help to detect the presence of leakage current electrons within a pixel during a "reference" acquisition, whose presence may be confirmed or disproved during subsequent reference acquisitions and can later be subtracted from the "final" acquisition data, thus combining the benefits of a fast readout rate without the drawback of increased noise hit rate. This feature, however, is mainly interesting in the case of relatively high initial noise values.

For this sensitivity studies we assume a default threshold of $Q=2 e^{-}$; in addition we also study the expected sensitivity for a threshold of $Q=1 e^{-}$and $Q=3 e^{-}$. The impact of background events from the leakage current is crucial and is subject to detailed device studies planned for the future. 
4.2 Simulation of background contributions from intrinsic radioactivity

In [10] a limit is derived for a background free experiment, while for this study we will discuss in addition the influence of background on the sensitivity of the experiment. Background from radioactive decays can be subdivided in two different categories, intrinsic background and background from external sources. We assume the shielding from external background sources to be very efficient so that remaining external backgrounds create surface events which can be rejected to a large extend, similar to the procedure used for detecting dark matter with semiconductor devices [21].

An irreducible background from internal radioactive decays is expected. The sensitive detector elements consist mainly of silicon and previous studies indicate, that the decay of ${ }^{32} \mathrm{Si}$ is expected to be the leading contribution to the internal background [22]. Cosmogenic activation of Si can produce the unstable isotope ${ }^{32} \mathrm{Si}$, which decays via $\beta^{-}$-decay with a half-life of $t_{1 / 2}=153$ year and an energy release of $227.2 \mathrm{keV}$. The decay leads to an energy deposition in the sensor and generates background events in the region of interest. The decay product ${ }^{32} \mathrm{P}$ is also unstable and decays with a half-life of $t_{1 / 2}=14.268$ days and an energy release of $1.711 \mathrm{MeV}$ to the stable isotope ${ }^{32} \mathrm{~S}$. A further cosmogenic background is ${ }^{3} \mathrm{H}$ produced via muon spallation and inelastic scattering of neutrons on silicon [28,29]. It undergoes a $\beta^{-}$-decay into the stable ${ }^{3} \mathrm{He}$ with an energy release of $18.592 \mathrm{keV}$.

We simulate the energy deposition in silicon of the ${ }^{32} \mathrm{Si}$ and the subsequent ${ }^{32} \mathrm{P}$ decay with the GEANT4 simulation package in version $10.2 \mathrm{p} 1$ using mostly the default processes described by the "Low Energy Electromagnetic Physics Working Group" [23-25]. Only the size of the sampling bins of the $\beta^{-}$spectra are decreased by a factor 100 relative to the default settings to increase the precision at lowest energies. We model a silicon only device with the geometry similar to the device to be used for initial dark matter searches. We set the activity of ${ }^{32} \mathrm{Si}$ in the sensor to $80 \mathrm{~kg}^{-1} \mathrm{day}^{-1}$ [22]. The decaying ${ }^{32} \mathrm{Si}$ isotopes are randomly distributed in the sensor. We explicitly note, since ${ }^{32} \mathrm{Si}$ is generated via cosmogenic activation, that the activity strongly depends on the time the silicon device is exposed to cosmic rays and the activity might vary for other devices.

To our knowledge no measurement of the cosmogenic ${ }^{3} \mathrm{H}$ production rate $R_{3 \mathrm{H}}$ in $\mathrm{Si}$ exists. Therefore, we rely on the simulation study [29] which found a strong dependence of $R_{3 \mathrm{H}}$ on the used simulation code, resulting in values ranging from 27.29 to $108.74 \mathrm{~kg}^{-1} \mathrm{day}^{-1}$. In a conservative approach we use the upper limit and set $R_{3 \mathrm{H}}=108.74 \mathrm{~kg}^{-1} \mathrm{day}^{-1}$. The cosmogenic induced activity $A_{3 \mathrm{H}}$ is then given by [28]

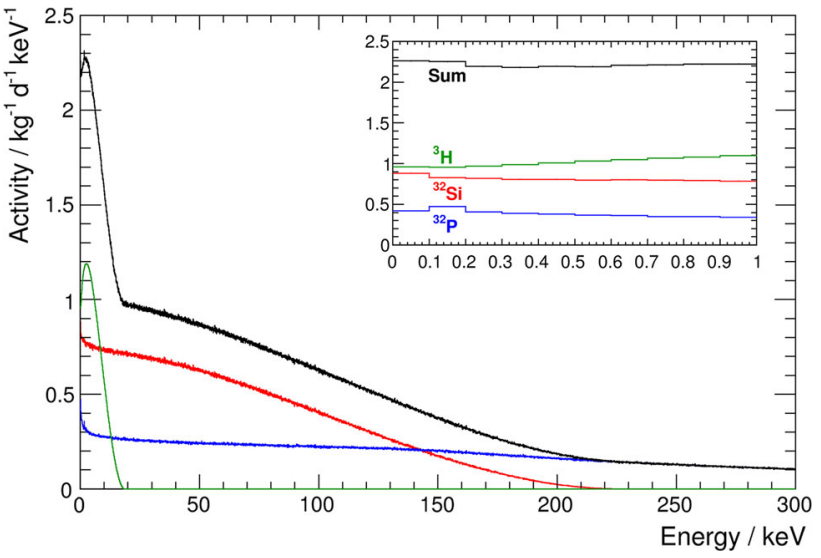

Fig. 10 Simulation of the energy deposition in a silicon sensor from radioactive ${ }^{32} \mathrm{Si} /{ }^{32} \mathrm{P}$ decays with a given activity of $80 \mathrm{~kg}^{-1}$ day ${ }^{-1}$ and ${ }^{3} \mathrm{H}$ decays with a given activity of $11.57 \mathrm{~kg}^{-1} \mathrm{day}^{-1}$. The histograms show the energy depositions from: the ${ }^{32} \mathrm{Si}$ decays (red), the subsequent ${ }^{32} \mathrm{P}$ decay (blue), the ${ }^{3} \mathrm{H}$ decay (green), and the sum of all decays (black). The inset zooms to the flat part of the spectrum below $1 \mathrm{keV}$. Statistical uncertainties are comparable to the line width

$A_{3 \mathrm{H}}=R_{3 \mathrm{H}} \cdot\left(1-e^{\frac{-\ln 2 \cdot \operatorname{texp}}{t_{1 / 2}}}\right) \cdot e^{\frac{-\ln 2 \cdot t_{\text {cool }}}{t_{1 / 2}}}$

where $t_{1 / 2}=12.32$ years is the half-life of ${ }^{3} \mathrm{H}, t_{\exp }$ is the period of exposure to cosmic rays, and $t_{\text {cool }}$ is the cooling time, i.e. the duration at an underground location. Assuming a duration of $t_{\exp }=2$ years between growth of the Si crystal and movement of the assembled detector to an underground laboratory, and afterwards an immediate start of operation, i.e. $t_{\text {cool }}=0$ years, results in $A_{3 \mathrm{H}}=11.57 \mathrm{~kg}^{-1} \mathrm{day}^{-1}$.

The simulated spectrum of the deposited energy in silicon from ${ }^{32} \mathrm{Si},{ }^{32} \mathrm{P}$, and ${ }^{3} \mathrm{H}$ decays is shown in Fig. 10. The simulation returns a flat spectrum with an activity of $\leq 2.26 \mathrm{~kg}^{-1} \mathrm{day}^{-1} \mathrm{keV}^{-1}$, corresponding to 0.825 $\mathrm{kg}^{-1}$ year ${ }^{-1} \mathrm{eV}^{-1}$, for energy depositions below $1 \mathrm{keV}$.

The RNDR DEPFET detector is able to detect single electrons with a resolution of $0.2 e^{-}$. To estimate the total background rate we follow the conversion from the total deposited energy to ionization as used in [10] and summarised in Eq. 2. We define as the signal region the energy range between the band gap energy of silicon $(1.1 \mathrm{eV})$ and the minimum energy needed to generate three electrons $(8.3 \mathrm{eV})$, corresponding to the first two bins in Fig. 1. By defining the first $Q$-bin as part of the signal region we follow a conservative approach and allow upward fluctuations of $Q=1$ to $Q=2$ hits, generated by the leakage current. With the given background activity of $0.825 \mathrm{~kg}^{-1}$ year ${ }^{-1} \mathrm{eV}^{-1}$ for energy depositions below $1 \mathrm{keV}$, as reported above, we expect a background rate of $5.94 \mathrm{~kg}^{-1}$ year $^{-1}$ in the region of $Q=1$ to $Q=2$. For the sensitivity studies we use a background rate of $6 \mathrm{~kg}^{-1}$ year ${ }^{-1}$. 


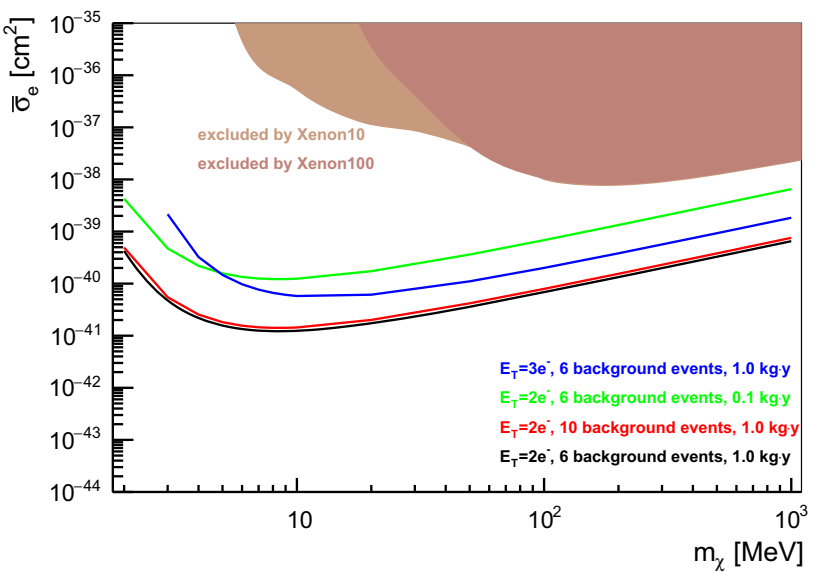

Fig. 11 Expected sensitivity for dark matter-electron scattering assuming a threshold of $Q=2 e^{-}$, six background events and an exposure of $1 \mathrm{~kg}$ year (black line). In addition we show the sensitivity with an increased threshold of $Q=3 e^{-}$(blue), reduced exposure of $0.1 \mathrm{~kg}$ year (green) and increased background of ten events (red). We assume a constant form factor of $F_{\mathrm{DM}}(q)=1$. For comparison the best limit using data from Xenon 10 and Xenon100 is shown [27]

\subsection{Expected sensitivity for detecting $\mathrm{MeV}$ dark matter with DETFET-RNDR detectors}

We use the number of predicted background events from ${ }^{32} \mathrm{Si},{ }^{32} \mathrm{P}$, and ${ }^{3} \mathrm{H}$ decays together with the code QEdark code to calculate the expected sensitivity of the experiment [10]. We consider a constant form factor of $F_{\mathrm{DM}}(q)=1$ only. We determine the expected sensitivity assuming six background events, an exposure of $1 \mathrm{~kg}$ year and a threshold of $Q=2 e^{-}$. We use the statistical approach described in [26] to determine the expected sensitivity. We assign no uncertainty to the number of expected background events and we take the number of observed events to be equal to the number of background events. The upper limit for the number of signal events for six background events is 6.75 events (95\% C.L.).

The expected sensitivity for different assumptions is shown in Figs. 11 and 12. Please note, that we assume in all cases no background events from leakage current events. With the default assumption of an energy threshold of two electrons, six background events and an exposure of $1 \mathrm{~kg}$ year we can reach a sensitivity of about $\bar{\sigma}_{\mathrm{e}}=10^{-41} \mathrm{~cm}^{2}$ for dark matter particles with a mass of $10 \mathrm{MeV}$. Assuming six background events the maximal sensitivity can be reached with an exposure of about $1 \mathrm{~kg}$ year, an exposure of 3 years improves the sensitivity only marginally. Increasing the threshold from two electrons to three electrons reduces the sensitivity in the $\mathrm{MeV}$ mass region by almost one order of magnitude. A reduction of the exposure to $0.1 \mathrm{~kg}$ year will lead to a similar loss in sensitivity.

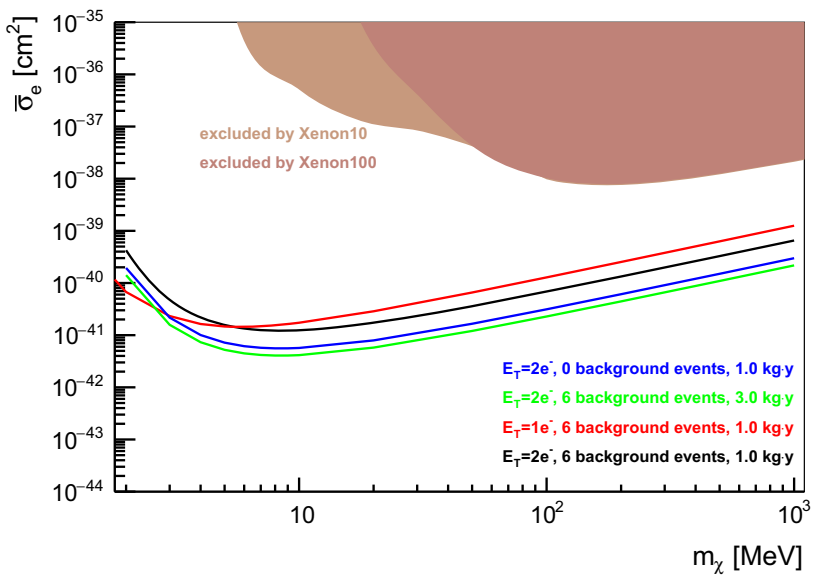

Fig. 12 Expected sensitivity for dark matter-electron scattering assuming a threshold of $Q=2 e^{-}$, six background events and an exposure of $1 \mathrm{~kg}$ year (black line). In addition we show the sensitivity with a reduced threshold of $Q=1 e^{-}$(red), increased exposure of $3 \mathrm{~kg}$ year (green) and decreased background of zero events (blue). We assume a constant form factor of $F_{\mathrm{DM}}(q)=1$. For comparison the best limit using data from Xenon10 and Xenon100 is shown [27]

\section{Conclusion}

The quest for particle dark matter is among the most urgent open topics of modern physics. The mass range for dark matter candidates, as well as the interaction rate with ordinary matter, is unpredicted. The parameter space for light dark matter in the $\mathrm{MeV}$ mass range still has some experimentally unexplored regions. We discuss the possibility to use a silicon detector operated as a RNDR DEPFET device to detect single electrons being produced by possible dark matter-electron scatterings. Measurements using a RNDR DEPFET prototype return an effective noise of $0.18 e^{-}$RMS, allowing to resolve single electrons. Assuming six background events in the signal region, a threshold of two electrons and an exposure of $1 \mathrm{~kg}$ year, we determine the expected sensitivity to be about $\bar{\sigma}_{\mathrm{e}}=10^{-41} \mathrm{~cm}^{2}$ for dark matter particles with a mass of $10 \mathrm{MeV}$.

Open Access This article is distributed under the terms of the Creative Commons Attribution 4.0 International License (http://creativecomm ons.org/licenses/by/4.0/), which permits unrestricted use, distribution, and reproduction in any medium, provided you give appropriate credit to the original author(s) and the source, provide a link to the Creative Commons license, and indicate if changes were made. Funded by SCOAP ${ }^{3}$.

\section{References}

1. G. Bertone, D. Hooper, J. Silk, Phys. Rep. 405, 279 (2005)

2. H. Baer, K.Y. Choi, J.E. Kim, L. Roszkowski, Phys. Rep. 555, 1 (2015)

3. D.B. Kaplan, Phys. Rev. Lett. 68, 741 (1992) 
4. K. Petraki, R.R. Volkas, Int. J. Mod. Phys. A 28, 1330028 (2013)

5. D.E. Kaplan, M.A. Luty, K.M. Zurek, Phys. Rev. D 79, 115016 (2009)

6. C. Boehm, P. Fayet, Nucl. Phys. B 683, 219 (2004)

7. C. Boehm, T.A. Ensslin, J. Silk, J. Phys. G 30, 279 (2004)

8. J.L. Feng, J. Kumar, Phys. Rev. Lett. 101, 231301 (2008)

9. R. Essig, J. Mardon, T. Volansky, Phys. Rev. D 85, 076007 (2012)

10. R. Essig, M. Fernandez-Serra, J. Mardon, A. Soto, T. Volansky, T.T. Yu, JHEP 05, 046 (2016)

11. P.W. Graham, D.E. Kaplan, S. Rajendran, M.T. Walters, Phys. Dark Univ. 1, 32 (2012)

12. S.K. Lee, M. Lisanti, S. Mishra-Sharma, B.R. Safdi, Phys. Rev. D 92(8), 083517 (2015)

13. J. Kemmer, G. Lutz, Nucl. Instrum. Methods A 253, 365 (1987)

14. S. Ott, A. Bähr, T. Brand, T. Dauser, N. Meidinger, M. Plattner, W. Stechele, JINST 11(01), C01028 (2016)

15. C. Marinas, JINST 10(11), C11002 (2015)

16. J. Schieck, Nucl. Instrum. Methods A 732, 160 (2013)
17. W. Treberspurg et al., in Proceedings of SPIE-The International Society for Optical Engineering, vol. 9905, ed. by J.W.A. den Herder, T. Takahashi, M. Bautz (2016), p. 99052C

18. S. Wolfel et al., IEEE Trans. Nucl. Sci. 54(4), 1311 (2007)

19. A. Bähr, Experimenteller Betrieb neuartiger DEPFETRNDR-Detektoren und mathematische Beschreibung der Mehrfachauslese. Diploma thesis, FH München (2010)

20. W. Treberspurg et al., Proc.SPIE 10397, 10397 (2017)

21. A. Aguilar-Arevalo et al., Phys. Rev. D 94(8), 082006 (2016)

22. A. Aguilar-Arevalo et al., JINST 10(08), P08014 (2016)

23. J. Allison et al., Nucl. Instrum. Methods A 835, 186 (2016)

24. J. Allison et al., IEEE Trans. Nucl. Sci. 53, 270 (2006)

25. S. Agostinelli et al., Nucl. Instrum. Methods A 506, 250 (2003)

26. G.J. Feldman, R.D. Cousins, Phys. Rev. D 57, 3873 (1998)

27. R. Essig, T. Volansky, T.T. Yu, Phys. Rev. D 96(4), 043017 (2017)

28. W.-Z. Wei, D.-M. Mei, C. Zhang, Astropart. Phys. 96, 24 (2017)

29. C. Zhang et al., Astropart. Phys. 84, 62 (2016) 